\title{
Efecto del ibuprofeno en el establecimiento de la gestación de embriones bovinos producidos in vitro
}

\author{
Effect of ibuprofen on the establishment of the gestation of in vitro \\ produced bovine embryos
}

\author{
Héctor Javier Narváez Bedoya ${ }^{1,2}$
}

\section{Resumen}

\begin{abstract}
El objetivo del estudio fue evaluar el efecto de la administración de ibuprofeno en el establecimiento de la gestación de embriones bovinos producidos in vitro. Se utilizaron 85 novillas Bos taurus x B. indicus como receptoras de embriones, las cuales fueron sometidas a un protocolo de sincronización del estro y de la ovulación con la inserción de un implante intravaginal de progesterona $\left(\right.$ Sincrogest $\left.{ }^{\circledR}\right)$ por ocho días, más la aplicación de $2 \mathrm{mg}$ de benzoato de estradiol (Sincrodiol ${ }^{\circledR}$ ). A la remoción del implante se aplicaron $500 \mu \mathrm{g}$ cloprostenol sódico (Sincrocio ${ }^{\circledR}$ ), 300 UI de gonadotrofina coriónica equina $\left(\right.$ Sincro eCG ${ }^{\circledR}$ ), más la administración de $1 \mathrm{mg}$ de cipionato de estradiol (SincroCP ${ }^{\circledR}$ ). Una hora antes a la transferencia de los embriones se aplicó $5 \mathrm{mg} / \mathrm{kg}$ de ibuprofeno IM. No se observó diferencia significativa en la tasa de gestación del grupo control (47.5\%) y del grupo ibuprofeno (35.5\%). Se concluye que la administración de ibuprofeno en novillas $B$. taurus $\mathrm{x}$ B. indicus una hora antes de la transferencia de los embriones in vitro no presentó efecto sobre el establecimiento de la gestación.
\end{abstract}

Palabras clave: antiinflamatorios no esteroideos, bovinos, desempeño reproductivo, prostaglandinas, transferencia de embriones

\section{Abstract}

The aim of this study was to evaluate the effect of the administration of ibuprofen in the establishment of the gestation of bovine embryos produced in vitro. In total, 85 Bos taurus $\mathrm{x}$ B. indicus heifers were used as embryo recipients, which were subjected to

\footnotetext{
${ }^{1}$ Universidad de Santander, Facultad de Ciencias Exactas, Naturales y Agropecuarias, Programa de Medicina Veterinaria, Grupo de Investigación en Ciencias Agropecuarias - GICA, Bucaramanga, Colombia

${ }^{2}$ E-mail: h.narvaez@mail.udes.edu.co
}

Recibido: 14 de mayo de 2020

Aceptado para publicación: 10 de enero de 2021

Publicado: 24 de abril de 2021 
a protocol of synchronization of oestrus and ovulation with the insertion of an intravaginal progesterone implant (Sincrogest $\left.{ }^{\circledR}\right)$ for eight days, plus the application of $2 \mathrm{mg}$ of oestradiol benzoate (Sincrodiol $\left.{ }^{\circledR}\right)$. To the removal of the implant, $500 \mu \mathrm{g}$ sodium cloprostenol (Sincrocio ${ }^{\circledR}$ ), 300 IU of equine chorionic gonadotropin (Sincro eCG ${ }^{\circledR}$ ), plus the administration of $1 \mathrm{mg}$ of oestradiol cypionate (SincroCP $\AA$,) were applied. One hour before embryo transfer, $5 \mathrm{mg} / \mathrm{kg}$ of ibuprofen IM was applied. No significant difference was observed in the gestation rate of the control group (47.5\%) and the ibuprofen group (35.5\%). It is concluded that the administration of ibuprofen in B. taurus $\mathrm{x}$ B. indicus heifers one hour before the transfer of the embryos in vitro had no effect on the establishment of gestation.

Keywords: non-steroidal anti-inflammatory, bovine, reproductive performance, prostaglandins, embryo transfer

\section{INTRODUCCIÓN}

Las pérdidas de la gestación por mortalidad embrionaria es uno de los factores de mayor impacto económico en los sistemas de producción de bovinos de leche y de carne (Santos et al., 2004). Esta problemática varía entre rebaños. Pudiendo ser tan baja como $3.2 \%$ (Silke et al., 2002) y llegar hasta $42.7 \%$ (Cartmill et al., 2001). Un trabajo de metaanálisis determinó que entre 50 y 44\% de la mortalidad embrionaria presentada en bovinos se encuentra en razas Bos indicus y la diferencia en razas Bos taurus (Reese et al., 2020).

Durante la gestación temprana, el embrión y el medio ambiente uterino establecen una interacción a través del mecanismo de reconocimiento materno de la gestación mediado por la síntesis del interferón-tau $\left(\mathrm{IFN}_{-}\right)$, proteína trofoblástica que tiene como función inhibir la luteolisis que es inducida por la secreción pulsátil de la $\mathrm{PGF}_{2 \alpha}$ (Demmers et al., 2001). No obstante, si se presenta una baja secreción de progesterona, las células trofoblásticas no tendrán la capacidad de producir eficientemente el IFN- T $_{T}$ conllevando de esta manera, a la síntesis de la $\mathrm{PGF}_{2 \alpha}(\mathrm{Mann}$ y Lamming, 2001). Varias estrategias antiluteoliticas han sido desarrolladas a fin de preservar el desarrollo embrionario temprano, siendo una de estas estrategias relacionada con la disminución de la respuesta luteolítica para proteger la sobrevivencia del embrión (Binelli et al., 2001).

Basado en esta hipótesis, algunos estudios han utilizado los antiinflamatorios no esteroideos como potentes inhibidores de la actividad de la enzima ciclooxigenasa 2 (COX-2), enzima que tiene la función de catalizar y de transformar a los precursores primarios de las prostaglandinas (PGHS-1 y PGHS-2) en PGF $_{2 \alpha}$ (Radi y Khan, 2006). La síntesis de la $\mathrm{PGF}_{2 \alpha}$ es regulada por un complejo intracelular endometrial y tiene la función de desencadenar la regresión del cuerpo lúteo $(\mathrm{CL})$ e inicio de un nuevo ciclo estral (Odensvik et al., 1989). La administración de antiinflamatorios previo a la transferencia de los embriones permite proteger la funcionalidad del CL, además de proporcionar mejor viabilidad embrionaria, lo que conlleva al bloqueo de la liberación precoz de la PGF $_{2 \alpha}$ después de la transferencia embrionaria (Guzeloglu et al., 2007). Por esta razón, el objetivo del presente estudio fue evaluar el efecto del ibuprofeno en el establecimiento de la gestación de embriones bovinos producidos in vitro. 


\section{Materiales y Métodos}

En la ejecución del estudio se dio cumplimiento a los aspectos mencionados el Art. 87 del Estatuto Nacional de Protección de los Animales (Resolución 008430 de 4 de octubre de 1993) (Garcés y Giraldo, 2012).

\section{Animales}

Fueron utilizadas 85 novillas (Bos taurus $\mathrm{x} B$. indicus) como receptoras de embriones, con edad y peso promedio de 24 meses y $350 \mathrm{~kg}$, respectivamente. Condición corporal de 4.0 (escala de $0-5$ puntos) y tracto reproductivo normal a nivel estructural y funcional. El estudio fue desarrollado bajo condiciones tropicales, con pasturas a base de Brachiaria decumbens Stapf y Brachiaria brizantha (Hochst. ex A. Rich.) Stapf (Poaceae), con suplementación mineral, agua ad libitum y sin suplementación balanceada.

\section{Manejo Experimental}

Se aplicó a las novillas un implante intravaginal de progesterona (P4) de $1 \mathrm{~g}$ (Sincrogest $^{\mathbb{}}$, Ouro Fino, Brasil) por 8 días, más la aplicación de $2 \mathrm{mg}$ de benzoato de estradiol (Sincrodiol ${ }^{\circledR}$, Ouro Fino, Brasil). A la remoción del dispositivo de $\mathrm{P} 4$ se aplicaron $500 \mu \mathrm{g}$ de cloprostenol sódico (Sincrocio ${ }^{\circledR}$, Ouro Fino, Brasil), 300 UI de gonadotrofina coriónica equina (Sincro eCG ${ }^{\circledR}$, Ouro Fino, Brasil), más la administración de $1 \mathrm{mg}$ de cipionato de estradiol (SincroCP ${ }^{\circledR}$ Ouro Fino, Brasil).

Nueve días después de la remoción del dispositivo de $\mathrm{P} 4$, se realizó la transferencia de embriones en tiempo fijo. Para esto, se hizo una ecografía transrectal (Mindray, DP 2200 VET, China) en las novillas para verificar la respuesta al protocolo hormonal con la presencia de CL. Fueron consideradas como receptoras de embriones aquellas con $\mathrm{CL} \geq 15$ $\mathrm{mm}$ de diámetro. Los embriones utilizados fueron a fresco y de origen in vitro en fase blastocisto y blastocisto expandido. Para la transferencia de los embriones, se aplicó anestesia epidural baja a las receptoras (Roxicaina ${ }^{\circledR} 2 \%$, Ropsohn, Colombia), además de una limpieza del recto e higienización de los genitales externos con agua y alcohol al $70 \%$. Los embriones fueron transferidos en el cuerno uterino ipsilateral al ovario con cuerpo lúteo. El diagnóstico de la gestación se hizo 60 días después de la transferencia de embriones, mediante ecografía transrectal utilizando un transductor linear de 5.0/7.5 MHz.

\section{Grupos Experimentales}

Las receptoras fueron localizadas en dos tratamientos: grupo control (no tratado) y grupo tratado con ibuprofeno, con aplicación IM del antiinflamatorio no esteroideo ibuprofeno a dosis terapéutica de $5 \mathrm{mg} / \mathrm{kg}$, una hora antes de la transferencia de los embriones. Este tiempo fue considerado como adecuado para la absorción y la estabilización de los niveles sanguíneos requeridos.

\section{Análisis Estadístico}

Se utilizó un delineamiento en bloques casualizados. Los dados fueron analizados utilizando análisis de varianza. En el caso de diferencias significativas fue utilizado el test de Chi-cuadrado, considerándose un nivel de significancia del 5\%.

\section{Resultados y Discusión}

Las tasas de gestación para el grupo control y para el grupo ibuprofeno fueron de $47.5 \%(19 / 40)$ y $35.5 \%(16 / 45)$, respectivamente (Figura 1), sin diferencias significativas entre grupos.

En bovinos, la mortalidad embrionaria es considerada una de las mayores problemáticas que enfrenta la industria ganadera. El mecanismo inicial de la gestación que se inicia con la fertilización alcanza aproximadamente el $90 \%$ en razas $B$. taurus de carne (Reese et al., 2020) con niveles entre $55.3 \mathrm{y}$ $87.8 \%$ en vacas lactantes y entre 59 y $98 \%$ 


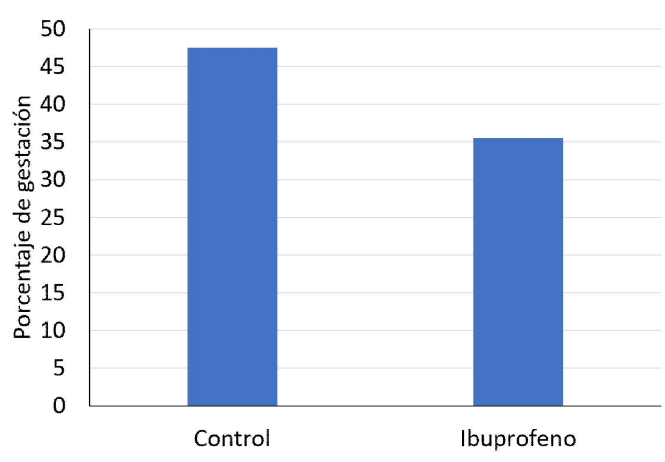

Figura 1. Efecto de la administración de ibuprofeno en el porcentaje de novillas (Bos taurus x Bos indicus) gestantes por la transferencia de embriones producidos in vitro

en vacas no lactantes (Santos et al., 2004), habiendo una diversidad de factores relacionados con fallas en la gestación en los primeros siete días de la concepción (Reese et al., 2020).

El ibuprofeno, como potente antiinflamatorio no esteroideo, tiene como función inhibir la síntesis de las prostaglandinas (Breuhaus et al., 1999), además de ser considerado un promotor angiogénico (Menzel et al., 1999) y un modificador de la síntesis de las citocinas y otras moléculas importantes para la función endometrial (Elli et al., 2001).

En estudios que utilizaron el ibuprofeno como promotor de la eficiencia reproductiva se observó que su administración una hora antes a la transferencia de los embriones tuvo un impacto altamente positivo en las tasas de gestación de embriones in vivo y criopreservados, respecto al grupo no tratado (82 vs $56 \%$, respectivamente, $\mathrm{p}<0.05$ ) (Elli et al., 2001). No obstante, estos resultados difieren de los hallados en el presente estudio donde no hubo diferencias significativas entre grupos; sin embargo, el presente grupo de autores (Narváez et al., 2010) reportaron una diferencia de $27.3 \%$ con el uso de ibuprofeno en las tasas de gestación de novillas de la raza Nelore transferidas con embriones congelados de origen in vivo.

Es importante resaltar que la transferencia de embriones de origen in vitro son generalmente de grado 1 (excelentes) y pueden llegar a ser estructuras no dependientes de los antiinflamatorios para llevar a cabo un adecuado mecanismo de la implantación y del mantenimiento de la gestación. Por otro lado, a veces se utiliza embriones grado 2 de calidad, siendo estos embriones considerados con menor potencial de desarrollo, lo que puede llegar a justificar el uso de los antiinflamatorios con la finalidad de evitar los efectos deletéreos de la $\mathrm{PGF}_{2 \alpha}$ si es liberada anticipadamente. Según, Scenna et al. (2005) la $\mathrm{PGF}_{2 \alpha}$, al entrar en contacto con el embrión, retarda o bloquea el desarrollo embrionario, disminuyendo la habilidad de transición de estructura pre-compacta a compacta (16 para 32 células), afectando de forma significativa las tasas de implantación y de gestación.

Erdem y Guzeloglu (2010) identificaron que la administración de meloxicam por vía subcutánea 15 días después de la inseminación artificial, inhibe la actividad del precursor de las prostaglandinas (PGHS-2), afectando de manera significativa el mecanismo de pre-implantación y subsecuentemente las tasas de gestación. Un estudio similar reportó que la aplicación de flunixin meglumine y carprofeno después de la inseminación artificial no incrementa las tasas de preñez en bovinos (von Krueger y Heuwieser, 2010). Recientemente, Kasimanickam et al. (2018) evaluaron el efecto del flunixin meglumine en receptoras de embriones de temperamento fuerte, indicando que estas hembras presentan altos niveles circulantes de isoprostano 8 -epi $\mathrm{PGF}_{2 \alpha}$ y de la sustancia $\mathrm{P}$, sustancias que tienen la capacidad de incrementar la producción de especies reactivas de oxígeno, siendo estas a su vez consideradas desfavorables para el medio ambiente uterino y con impacto negativo sobre las tasas de gestación. 


\section{Conclusiones}

La administración de ibuprofeno en novillas $B$. taurus $\times$ B . indicus una hora antes de la transferencia de los embriones no presentó un efecto beneficioso sobre el establecimiento de la gestación de embriones producidos in vitro.

\section{Agradecimientos}

Los autores agradecen a la Ganadería Santa Isabella, Sabana de Torres, Santander, Colombia, por facilitar los animales para la ejecución del proyecto.

\section{Literatura Citada}

1. Binelli $M$, Thatcher $W W$, Mattos $R$, Baruselli PS. 2001. Antiluteolytic strategies to improve fertility in cattle. Theriogenology 56: 1451-1463. doi: 10.1016/s0093-691x(01)00646-x

2. Breuhaus BA, DeGraves FJ, Honore EK, Papich MG. 1990. Pharmacokinetics of ibuprofen after intravenous and oral administration and assessment of safety of administration to healthy foals. Am J Vet Res 60: 1066-1073

3. Cartmill JA, El-Zarkouny SZ, Hensley BA, Rozell TG, Smith JF, Stevenson JS. 2001. An alternative AI breeding protocol for dairy cows exposed to elevated ambient temperatures before or after calving or both. J Dairy Sci 84: 799806. doi: 10.3168/jds.S0022-0302(01)74536-5

4. Demmers KJ, Derecka K, Flint A. 2001. Trophoblast interferon and pregnancy. Reproduction 121: 41-49. doi: 10.1530/rep.0.1210041

5. Elli $M$, Gaffuri $B$, Frigerio $A$, Zanardelli M, Covini D, Candiani M, Vignali M. 2001. Effect of a single dose of ibuprofen lysinate before embryo transfer on pregnancy rates in cows. Reproduction 121:151-154
6. Erdem H, Guzeloglu A. 2010. Effect of meloxicam treatment during early pregnancy in Holstein heifers. Reprod Domest Anim 45: 625-628. doi: 10.1111/ j.1439-0531.2008.01317.x

7. Garcés LF, Giraldo C. 2012. Bioética en la experimentación científica con animales: cuestión de reglamentación o de actitud humana. Rev Lasallista Investig 9: 159-166.

8. von Krueger X, Heuwieser W. 2010. Effect of flunixin meglumine and carprofen on pregnancy rates in dairy cattle. J Dairy Sci 93: 5140-5146. doi: 10.3168/jds.2010-3072

9. Kasimanickam RK, Hall JB, Estill CT, Kastelic JP, Joseph C, Abdel Aziz $R L$, et al. 2018. Flunixin meglumine improves pregnancy rate in embryo recipient beef cows with an excitable temperament. Theriogenology 107: 7077. doi: 10.1016/j.theriogenology.2017.10.043

10. Mann GE, Lamming GE. 2001. Relationship between maternal endocrine environment, early embryo development and inhibition of the luteolytic mechanism in cows. Reproduction 121: 175-180. doi: 10.1530/ rep. 0.1210175

11. Menzel EJ, Burtscher H, Kolarz G. 1999. Inhibition of cytokine production and adhesion molecule expression by ibuprofen is without effect on transendothelial migration of monocytes. Inflammation 23: 275-286. doi: 10.1023/ a:1020230220971

12. Narváez HJ, Fontes RS, Costa RLD, Quirino CR, Moreira LZ. 2010. Efeito do ibuprofeno administrado uma hora antes da inovulação de embriões bovinos. Arq Bras Med Vet Zoo 62: 504-510. doi: 10.1590/S0102-09352010000300002

13. Odensvik K, Cort N, Basu S, Kindahl H. 1989. Effect of flunixin meglumine on prostaglandin F2 $\alpha$ synthesis and metabolism in the pig. J Vet Pharmacol Ther 12: 307-311. doi: 10.1111/j.13652885.1989.tb00676.x 
14. Radi ZA, Khan NK. 2006. Effects of cyclooxygenase inhibition on the gastrointestinal tract. Exp Toxicol Pathol 58: 163-173. doi: 10.1016/j.etp.2006.06.004

15. Reese ST, Franco GA, Poole RK, Hood R, Fernandez L, Oliveira RV, Cooke RF, et al. 2020. Pregnancy loss in beef cattle: a meta-analysis. Anim Reprod Sci 212: 106251. doi: 10.1016/ j.anireprosci.2019.106251

16. Santos JE, Thatcher WW, Chebel RC, Cerri RL, Galvão KN. 2004. The effect of embryonic death rates in cattle on the efficacy of estrus synchronization programs. Anim Reprod Sci 82-83: 513 535. doi: 10.1016/j.anireprosci.2004.04.015
17. Scenna FN, Hockett ME, Towns TM, Saxton AM, Rohrbach NR, Wehrman ME, Schrick FN. 2005. Influence of a prostaglandin synthesis inhibitor administered at embryo transfer on pregnancy rates of recipient cows. Prostag Oth Lipid M 78: 38-45. doi: 10.1016/j.prostaglandins.2005.02.003

18. Silke V, Diskin MG, Kenny DA, Boland MP, Dillon P, Mee JF, Sreenan JM. 2002. Extent, pattern and factors associated with late embryonic loss in dairy cows. Anim Reprod Sci 71: 1-12. doi: 10.1016/s0378-4320(02)00016-7

19. von Krueger X, Heuwieser W. 2010. Effect of flunixin meglumine and carprofen on pregnancy rates in dairy cattle. J Dairy Sci 93: 5140-5146. doi: 10.3168/jds.2010-3072 